Restricted Fourter series and Convergence of power series. 359

\title{
ON RESTRICTED FOURIER SERIES AND THE CONVERGENCE OF POWER SERIES
}

\author{
By Prof. W. H. Yor:NG.
}

[Received Octiober 4th, 1917.-Read December 6th, 1917.]

1. In a previous communication to the Society, ${ }^{*}$ I introduced the term "restricted Fourier series of the first, second, ... class," using the expression in the following sense :-

The $p$-th derived series of the Fourier series of $F(x)$ is said to be a restricted Fourier series of the $p$-th class, and to be restricted to one or more intervals $(\alpha, \beta)$, if, throughout each completely open interval in $(\alpha, \beta)$, $F(x)$ is a $p$-th integral.

The $p$-th differential coefficient of $F(x)$ in $(\alpha, \beta)$, defined almost everywhere, is then called the function associated with the restricted Fourier series.

The properties of a Fourier series retained by these trigonometrical series, in the interval or intervals to which they are restricted, were shewn in the memoir quoted to be, in general, what may be called "Cesàrosummation properties, index $p . "$ In particular, taking $p=1$, the restricted Fourier series of the first class may be defined as trigonometrical series whose integrated series are Fourier series which converge in every completely open interval in $(\alpha, \beta)$ to a function $F(x)$ which is an absolutely convergent integral; and the properties of Fourier series retained by these trigonometrical series are all those involving Cesàro convergence, index 1 . In particular the upper and lower functions, obtained by Cesàro summation $(C 1)$, at a point $x$ of the interval of restriction, are independent of the nature of the associated function, except in a neighbourhood of $x$ as small as we please.

In a subsequent note, $\dagger$ presented to the Royal Society, I actually ob-

- "On the Convergence of the Derived Series of Fourier Series" (1916).

† "On the Ordinary Convergence of Restricted Fourier Series" (1917), Proc. Roy. Soc., A, Vol. 93, pp. 276-292.

SER. 2. vor. 17 . No. 1324 . 
tained classes of restricted Fourier series which, without being themselves Fourier series, converged in the ordinary way throughout an interval. The method used was adapted ad hoc; I had, in fact, practical applications in view in the results I obtained. In the present communication my point of view is more theoretical and accordingly considerably wider in its consequences.

The point of departure is a remarkably simple theorem which I have been so fortunate as to discover. This theorem, while it more than justifies the introduction of the term " restricted Fourier series," and their separate consideration, seems to me, at the same time, to render desirable the distinction of a certain sub-class of restricted Fourier series as " ordinary restricted Fourier series," or, for brevity, "R.F. series."

Any trigonometrical series

$$
\sum_{n=1}^{\infty}\left\{a_{n} \cos n x+b_{n} \sin n x_{\}}\right.
$$

-where for simplicity we have omitted the constant term-is defined to be an ordinary restricted Fourier series, or R.F. series, if it verifies two conditions :

(i)

$$
a_{n} \rightarrow 0, \quad b_{n} \rightarrow 0 \quad(n \rightarrow \infty) ;
$$

(ii) the integrated series

$$
\sum_{n=1}^{\infty} \frac{1}{n}\left\{a_{n} \sin n x-b_{n} \cos n x\right\}
$$

converges to an absolutely convergent integral

$$
F(x)=\int f(x) d x,
$$

in the interval or intervals of restriction $(\alpha<x<\beta)$.

The function associated with the R.F. series is then

$$
f(x)=\frac{d F}{d x},
$$

and is, of course, only defined almost everywhere in $(\alpha, \beta)$.

We may use the symbolic notation

$$
f(x) \sim \sum_{n=1}^{\infty}\left\{a_{n} \cos n x+b_{n} \sin n x\right\} \quad(\alpha<x<\beta),
$$


to denote that the series on the right is an R.F. series, with $(\alpha, \beta)$ as interval of restriction of $f(x)$ for associated function.*

The condition (i) is well-known to be verified by a Fourier series. Thus R.F. series include Fourier series. Also in virtue of (i), the squares of the coefficients of the integrated series form a convergent series, so that by the Riesz-Fischer theorem $t$ the integrated series is a Fourier series. Thus R.F. series are included in the class of restricted Fourier series of the first class.

The object of the new term R.F. series is not, however, merely to connect the class with the sub-class, but to emphasize the extensive analogy between the new series and Fourier series, and thus at once to assist the memory and to render possible a generalisation of known theorems. It is evident that, the closer the analogy, the greater will be the advantage obtained by the nomenclature, and the less the danger of compensating disadvantages, due to the involuntary assumption that theorems true for Fourier series are also true for the extended class of ordinary restricted Fourier series (R.F. series), when this is not the case. With the definition just given, almost all the more important properties of Fourier series are found to hold good for R.F. series in the interval or intervals to which we restrict ourselves. Such a notable extension of our knowledge is rendered possible by the fundamental theorem above referred to.

I have, in fact, been able to prove that the conditions for convergence and uniform convergence of an R.F. series in the interval of restriction are of precisely the same form as for a Fourier series. The same is true for the various possible forms of oscillation, uniform or otherwise.

Again the same is true for the Cesaro convergence of the derived series of R.F. series : the upper and lover functions of the p-th derived series of an R.F. series, when summed in the Cesiro manner $(C p)$, at any point internal to the interval of restriction, depend only on the nature of the associated function! in a neighbourhood of the point as small as we please.

- The symbol $\sim$ followed by $(-\pi<x<\pi)$ will then not be equivalent to the recognised symbol $\sim$ for a Fourier series, since the new symbolism does not even imply that the integrated series converges at the end-points $-\pi$ and $\pi$, or that, if it converges, the sum is other than discontinuous there, while $\sim$ implies both these facts, and also that $F(x)$ is an integral in the closed interval $(-\pi, \pi)$.

+ For a collection of the existing proofs of this theorem see W. H. Young and Grace Chisholm Young, "On the Riesz-Fischer 'Theorem," Quarterly Journal of Mathematics.

$\ddagger$ Which is, of course, the $p$-th differential coefficient of the function associated with the R.F. series from which our series is derived, since our series is a restricted Fourier series of the $(p+1)$-th class. 
The condition imposed on the coefficients of the trigonometrical series considered $-a_{n} \rightarrow 0, b_{n} \rightarrow 0$ for an R.F. series, $n^{-p} a_{n} \rightarrow 0, n^{-p} b_{n} \rightarrow 0$ for its $p$-th derived series-is one which not only ensures what is desired, but is also necessary when the series is required to converge in the manner considered throughout an interval. I have not thought it necessary to insist on the somewhat more general theorems which arise when convergence at a point is required. In this case the condition may be relaxed,

$$
\left(a_{n} \cos n x+b_{n} \sin n x\right) \rightarrow 0
$$

in the former case, and

$$
n^{-p}\left(a_{n} \cos n x+b_{n} \sin n x\right) \rightarrow 0
$$

in the latter case, being then sufficient, as well as, of course, necessary.

It is not merely in the region of convergence that the analogy between Fourier series and R.F. series is complete. It is obviously also complete in the case, for example, of the chain of theorems, based on Riemann's work, due to Lebesgue, Fejér, de la Vallée Poussin, and myself, on certain conditions under which a trigonometrical series may be asserted to be a. Fourier series. Nor do these theorems exhaust the list: only those in which the actual representation of the coefficients in terms of the associated function are required form exception.

The results to which we are led are not only important in the theory of trigonometrical series and its applications ; they lead, when interpreted in the language of the complex variable, to a notable extension of our knowledge of the behaviour of power series on their circle of convergence. Our main theorem shews, for example, that the uniform convergence of the power series $\Sigma c_{n} z^{\prime \prime}$, where $c_{n} \rightarrow 0(n \rightarrow \infty)$, can be asserted with confidence if (i) the sum-function has a unique limiting value at the point in ques. tion, and at the points of the circumference in its neighbourhood, and (ii) the function of $\theta$ formed by these limiting values on the circumference is a function of bounded variation.

As before we may change the condition $c_{n} \rightarrow 0$ into $n^{-n} c_{n} \rightarrow 0(n \rightarrow \infty)$, provided we replace the ordinary convergence by Cesàro convergence $(C p)$.

This theorem includes, as a very special corollary, Fatou's convergence theorem, the recognised importance of which in the Theory of Functions of a Complex Variable arises from the very fact that nothing is required to be known about the sum function, except in the neighbourhood of the point considered (the regularity, in fact, of the sum-function there): the only condition added is the necessary one $\left(c_{n} \rightarrow 0\right)$ for convergence.

The three proofs that have been given of Fatou's convergence theorem are sufficiently long and complicated; even the shortest, given by Marcel 
Riesz, is considerably more difficult than our proof of the more general theorem, based as it is only on the essential features of the problem, and accordingly almost intuitive.

In this connexion it may not be out of place to remark that inadequate use appears still to have been made hitherto of real function theory in dealing with the complex variable, owing partly perhaps to the mistrust of the older analysts in the theory of the real variable, as concerned with the pathology rather than the morphology of functions, and this though it has given to mathematics in the new concept of integration perhaps the greatest impetus since the time of Riemann.

2. The main theorem is as follows :-

Theовем 1.-The upper and lower functions of an R.F. series at a point $x$ internal to its interval of restriction $(\alpha, \beta)$, depend only on the properties of the associated function in a neighbourhood of $x$ as small as we please.

Let the R.F. series be

$$
f(x) \sim \sum_{n=1}^{\infty}\left\{a_{n} \cos n x+b_{n} \sin n x\right\} \quad(a<x<\beta),
$$

where, by definition, $\quad a_{n} \rightarrow 0, \quad b_{n} \rightarrow 0 \quad(n \rightarrow \infty)$.

The first integrated series will then be a Fourier series, say

$$
F(x) \sim \sum_{n=1}^{\infty}\left\{\frac{a_{n}}{n} \sin n x-\frac{b_{n}}{n} \cos n x\right\},
$$

where, in the interval of restriction,

$$
F(x)-F(a)=\int_{a}^{x} f(x) d x \quad(a<x<\beta) .
$$

The sum $s_{n}$ of $n$ terms of the series (1) is then obtained by differentiating the sum of $n$ terms of the series (3). Therefore

$$
\begin{aligned}
s_{n}= & \frac{1}{2 \pi} \frac{d}{d x} \int_{0}^{\pi}\{F(x+u)+F(x-u)\} \sin \left(n+\frac{1}{2}\right) u \operatorname{cosec} \frac{1}{2} u d u \\
= & \frac{1}{2 \pi} \frac{d}{d x} \int_{0}^{e}\{F(x+u)+F(x-u)\} \sin \left(n+\frac{1}{2}\right) u \operatorname{cosec} \frac{1}{2} u d u \\
& +\frac{1}{2 \pi} \frac{d}{d x} \int_{e}^{\pi}\{F(x+u)+F(x-u)\} \sin \left(n+\frac{1}{2}\right) u \operatorname{cosec} \frac{1}{2} u d u,
\end{aligned}
$$


where $e$ is chosen as small as we please, and certainly small enough for the interval $(x-e, x+e)$ to lie inside $(\alpha, \beta)$. The first of the two terms on the right in (5) depends only on the properties of $f(x)$ in a neighbourhood of $x$ as small as we please. Thus our theorem only requires us to prove the second term in (5) to tend towards zero when $n \rightarrow \infty$. We may write the second term in the form

$$
\frac{1}{2 \pi} \frac{d}{d x} \int_{e}^{\pi}\{F(x+u)+F(x-u)\} \sin n u \cot \frac{1}{2} u d u+\frac{1}{2 \pi} \gamma_{n},
$$

where $\quad \gamma_{n}=\gamma_{-n}=\frac{d}{d x} \int_{e}^{\pi}\{F(x+u)+F(x-u)\} \cos n u d u$,

and our theorem will be proved if we shew that the expression (6) tends. to zero when $n \rightarrow \infty$.

3. Now, since (3) is a Fourier series,

$$
\begin{aligned}
& \frac{d}{d x} \int_{0}^{\pi}\{F(x+u)+F(x-u)\} \cos n u d u=\pi\left\{a_{n} \cos n x+b_{n} \sin n x\right) \rightarrow 0 \\
& (n \rightarrow \infty) \text {, }
\end{aligned}
$$

by (2).

Also since, in $(\alpha, \beta), F(x)$ is the integiral of $f(x)$,

$\frac{d}{d x} \int_{0}^{e}\{F(x+u)+F(x-u)\} \cos u u d u=\int_{0}^{e}\{f(x+u)+f(x-u)\} \cos u u d u \rightarrow 0$ by the theorem of Riemann-Lebesgue.**

$$
(n \rightarrow \infty) \text {, }
$$

From (9) and (10), by subtraction,

$$
\gamma_{n} \rightarrow 0 \quad(n \rightarrow \infty) .
$$

Thus the condition to be proved becomes

$$
Q_{u}=\frac{d}{d x} \int_{e}^{\pi}\{F(x+u)+F(x-u)\} \sin u u \cot \frac{1}{2} u d u \rightarrow 0 \quad(u \rightarrow \infty) .
$$

4. Now let $\phi(u)$ denote an odd function having the following properties :-

(i) $\phi(u)=\cot \frac{1}{2} u$ in $(e, \pi)$, and in $(-e,-\pi)$;

(ii) $\phi(0)=0$;

* B. Riemann, Habilitationschrift, $§ 10$ (1854); H. Lebesgue, Leçons sur les séries trijonométriques (1906), p. 61. 
1917.] Regtricted Fourier series and convergence of power Series. 359

(iii) $\phi(u)$ is continuous at $u=e$ and $u=-e$;

(iv) $\phi(u)$ possesses its two* first differential coefficients at every point of the closed interval $(-e, e)$, and these are bounded.

This is clearly feasible, and the function $\phi(u)$ is then the integral of an integral. We have, therefore,

$$
\phi(u)=\sum_{p=1}^{\infty} c_{p} \sin p u,
$$

where the series converges uniformly. The first differentiated series also converges uniformly and its sum is $\phi^{\prime}(u)$.

The coefficients $c_{p}$ then have the property

$$
c_{p}=O\left(p^{-3}\right),
$$

and the series formed by them is absolutely convergent.

Let us write

$$
C=\sum_{1}^{\infty}\left|c_{p}\right|
$$

and

$$
K_{n}=\sum_{n}^{\infty}\left|c_{p}\right|
$$

so that

$$
K_{n} \rightarrow 0 \quad(n \rightarrow \infty)
$$

5. Let us write also

$$
G(x)-G(-\pi)=\int_{-\pi}^{x} F(x) d x \quad(-\pi \leqslant x \leqslant \pi) .
$$

We then have, integrating by parts,

$$
\begin{aligned}
\int_{e}^{\pi}\{F(x+u)+F(x-u)\} & \sin n u \phi(u) d u \\
= & -\{G(x+e)-G(x-e)\} \sin n e \phi(e) \\
& -n \int_{e}^{\pi}\{G(x+u)-G(x-u)\} \phi(u) \cos n u d u \\
& -\int_{e}^{\pi}\{G(x+u)-G(x-u)\} \phi^{\prime}(u) \sin n u d u .
\end{aligned}
$$

Now $F$ is the differential coefficient of $G$ at every point of the closed

* We can easily make as many of the differential coefficients exist as we please, or all is them : this is not required here, but will be required in $\S 9$. 
interval $(x-e, x+e)$ inside the interval of restriction $(\alpha, \beta)$, in which $F$, being an integral, is continuous : and, in $(e, \pi), F$ is almost everywhere equal to $d G / d x$. Therefore, differentiating (17), we get

$$
\begin{aligned}
Q_{n}= & -\{F(x+e)-F(x-e)\} \sin n e \phi(e)-n \int_{e}^{\pi}\{F(x+u)-F(x-u)\} \cos n u d u \\
& -\int_{e}^{\pi}\{F(x+u)-F(x-u)\} \phi^{\prime}(u) \sin n u d u .
\end{aligned}
$$

But since the Fourier series of $\phi$ and $\phi^{\prime}$ converge uniformly, we may replace these functions by their Fourier series and integrate term-by-term. Having.done this, we have only to transform back the expression under the sign of integration, $c_{p} \sin p u$ replacing $\phi(u)$, and we get

$$
\begin{aligned}
Q_{n} & ={ }_{p=1}^{\infty} \sum_{p=1}^{\infty} c_{p} \frac{d}{d x} \int_{e}^{\pi}\{F(x+u)-F(x-u)\} \sin n u \sin p u d u \\
& =\sum_{p=1}^{\infty} c_{p}\left\{\gamma_{p-n}-\gamma_{p+n}\right\},
\end{aligned}
$$

where $\gamma_{n}$ is given by (7) and satisfies (11).

6. Therefore whatever positive quantity $\epsilon$ we choose, we can find a corresponding integer $m$ such that, for all values of $r \geqslant m$,

Hence, when $n \geqslant m$,

$$
\left|\gamma_{r}\right|<\epsilon
$$

$$
\begin{gathered}
\left|\sum_{p=1}^{\infty} c_{p} \gamma_{p+n}\right| \leqslant \epsilon \sum_{p=1}^{\infty}\left|c_{p}\right| \leqslant \epsilon C, \\
\left|\sum_{p=n+m+1}^{\infty} c_{p} \gamma_{p-n}\right| \leqslant \epsilon \sum_{p=n+m+1}^{\infty}\left|c_{p}\right| \leqslant \epsilon K_{n} \rightarrow 0 \quad(n \rightarrow \infty) .
\end{gathered}
$$

Writing

$$
\begin{aligned}
\sum_{p=1}^{n+m} c_{p} \gamma_{p-n} & =\sum_{p=1}^{n-n} c_{p} \gamma_{n-p}+\sum_{p=n-n+1}^{n} c_{p} \gamma_{n-p}+\sum_{p=n+1}^{n+m} c_{p} \gamma_{p-n} \\
& =S_{1}+S_{2}+S_{3},
\end{aligned}
$$

we have

$$
\left|S_{1}\right|=\left|\sum_{p=1}^{n-m} c_{p} \gamma_{n-p}\right|=\left|\sum_{r=m}^{n-1} c_{n-r} \gamma_{r}\right| \leqslant \epsilon \sum_{p=1}^{n-m}\left|c_{p}\right| \leqslant \epsilon C .
$$

Also, if $M$ be any positive quantity, not less than the absolute values 
1917.] Restricted Fourier series and convergence of power series. 361 of the $m+1$ quantities $\gamma_{0}, \gamma_{1}, \ldots, \gamma_{m}$, we have

$$
\left|S_{2}\right|=\left|\sum_{p=n-m+1}^{n} c_{p} \gamma_{n-p}\right|=\left|\sum_{r=0}^{m-1} c_{n-r} \gamma_{r}\right| \leqslant M \sum_{p=m-n+1}^{n}\left|c_{p}\right| \leqslant M K_{n-m} \rightarrow \infty
$$

and

$$
\left|S_{8}\right|=\left|\sum_{p=n+1}^{n+m} c_{p} \gamma_{p-n}\right|=\left|\sum_{r=1}^{m} c_{n+r} \gamma_{r}\right| \leqslant M \sum_{p=n+1}^{n+m}\left|c_{p}\right| \leqslant M K_{n} \rightarrow 0,
$$

Using (21)-(25) in (19), we get

$$
(n \rightarrow \infty) \text {. }
$$

$$
\operatorname{Llt}_{n \rightarrow \infty}\left|Q_{n}\right| \leqslant 2 \epsilon C,
$$

whence, since $\epsilon$ is as small as we please,

$$
Q_{n} \rightarrow 0 \quad(n \rightarrow \infty)
$$

Thus the required condition is satisfied, which proves the theorem.

7. In the preceding proof we have only used the fact that

$$
a_{n} \cos n x+b_{n} \sin n x \rightarrow 0 \quad(n \rightarrow \infty),
$$

namely in (9), and not the whole assumption $a_{n} \rightarrow 0, b_{n} \rightarrow 0$.

If, however, we require convergence in a whole interval, whether $(\alpha, \beta)$, or a part of $(\alpha, \beta)$, the coefficients $a_{n}$ and $b_{n}$ themselves must, as Riemann already remarked,* tend towards zero. It is then easily seen that $Q_{n} \rightarrow 0$ uniformly.

Indeed, we can find $m$ independent of $x$, so that for $n \geqslant m$,

$$
\left|\frac{1}{\pi} \frac{d}{d x} \int_{0}^{\pi}\{F(x+u)+F(x-u)\} \cos n u d u\right| \leqslant\left|a_{n}\right|+\left|b_{n}\right| \leqslant \frac{1}{2} \epsilon .
$$

The uniformity of approach to zero of the corresponding integral whose upper limit of integration is $e$ instead of $\pi$ is part of the theorem of Riemann-Lebesgue. Hence

$$
\left|\gamma_{n}\right| \leqslant \epsilon \quad(n \geqslant m)
$$

$m$ being independent of $x$.

In $\S 6$ the quantity $M$ may be likewise chosen independent of $x$. In- 
deed, as in $\S 3$, introducing the argument $x$ explicitly,

$$
\begin{aligned}
\left|\gamma_{n}(x)\right| & =\left|\pi\left\{a_{n} \cos n x+b_{n} \sin n x\right\}-\int_{0}^{e}\{f(x+u)+f(x-u)\} \cos n u d u\right| \\
& \leqslant \pi\left\{\left|a_{n}\right|+\left|b_{n}\right|\right\}+\int_{x-e}^{x+e}|f(t)| d t .
\end{aligned}
$$

Putting $n$ in turn equal to $0,1, \ldots, m$, we see that we may put

$$
M=\pi \sum_{0}^{m_{2}}\left\{\left|a_{n}\right|+\left|b_{n}\right|\right\}+m \int_{a}^{\beta}|f(t)| d t,
$$

and then $M$ is, as it should be, not less than $\gamma_{0}(x), \gamma_{1}(x), \ldots, \gamma_{m}(x)$, wherever $x$ may be in $(\alpha, \beta)$.

By (19) and (21)-(25) we have therefore, $m$ and $M$ being independent of $x$,

$$
\mid Q_{n}(x) ! \leqslant 2 \epsilon C+{ }_{\epsilon} K_{n}+M K_{n-m}+M K_{n} \quad(n \geqslant m),
$$

which shews that $Q_{n}(x)$ converges uniformly to zero, and proves our required result.

8. As an immediate corollary to Theorem 1 , in conjunction with $\$ 7$, we have the following :-

Theorem 2.-If $f(x) \sim \Sigma\left\{a_{n} \cos n x+b_{n} \sin n x\right\} \quad(\alpha<x<\beta)$,

and $f_{1}(u)$ is any absolutely integrable* function, agreeing with $f(u)$ in $(x-e, x+e)$, inside $(\alpha, \beta)$, then the R.F. series (1) and the Fourier series of $f_{1}(u)$ have the same peculiarities with regard to convergence or oscillation, whether at the point $x$ or in the interval $(x-e, x+e)$.

Also in all integration theorems involving term-by-term integration of a Fourier series, when multiplied by an absolutely integrable function $g(x)$, we may substitute an R.F. series for the Fourier series, provided the range of integration lie within the corresponding interval of restriction.

Hence we have for R.F. series the same criteria of convergence as for Fourier series. In particular Dirichlet's criterion may be applied, and we have the following theorem :-

Theorem 3.-The R.F. series of a continuous function of bounded variation converges uniformly to that function.

9. As the first class of restricted Fourier series contains R.F. series, 
the second class will contain the derived series of R.F. series. These will evidently be characterised among the series of the second class by the condition

$$
n^{-1} a_{n} \rightarrow 0, \quad n^{-1} b_{n} \rightarrow 0 \quad(n \rightarrow \infty) .
$$

Generally the $p$-th derived series of R.F. series are characterised among the restricted Fourier series of the $(p+1)$-th class by the condition

$$
n^{-p} a_{n} \rightarrow 0, \quad n^{-n} b_{n} \rightarrow 0 \quad(n \rightarrow \infty) .
$$

In a precisely similar manner to that used in proving our main theorem, we can prove the following more general theorem applying to these last-mentioned series :-

'Theoren 4.-The Cesirn upper and lower functions (index $p$ ) of the $p$-th derived series of an R.F. series, at a point $x$ internal to its interval of restriction $(\alpha, \beta)$, depend only on the properties of the associated function in a neighbourlood of $x$ as small as we please.

It will be suficient to remark that, in the case when $p=1$, the discussion turns on the fact that, since $n^{-1} a_{n} \rightarrow 0, n^{-1} b_{n} \rightarrow 0$,

$$
\frac{1}{2 n \pi} \frac{d^{2}}{d x^{2}} \int_{e}^{\pi}\{G(x+u)+G(x-u)\} \sin ^{2} \frac{1}{2} n t \operatorname{cosec}^{2} \frac{1}{2} t d t \rightarrow 0 \quad(n \rightarrow \infty)
$$

-the approach to the limit being uniform in any interval internal to $(\alpha, \beta)$ -where $G(x)=\int_{a}^{x} d t \int f(t) d t$ is the function to which the second integrated series converges in $(\alpha, \beta)$.

This follows as before, bearing in mind that, when we replace $\sin ^{2} \frac{1}{2} n t$ by $\frac{1}{2}(1-\cos n t)$, the part of our expression independent of $\cos n t$ has the limit zero as in $\$ 3$, the theorem of Riemann-Lebesgue applying, since we may now differentiate twice under the sign of integration. The remaining part of our expression is treated precisely as in $\$ \S 4-6$, the function $\phi(u)$ being provided with the proper: array of differential coefficients. * The presence of the factor $1 / n$ in our expression ensures the adequacy of the condition $n^{-1} a_{n} \rightarrow 0, n^{-1} b_{n} \rightarrow 0$.

10. Finally it may be remarked that there are corresponding theorems for fractional Cesàro convergence, in which the Fourier series of a function of bounded variation takes the place of that of an integral. Readers of my previous papers will be able to supply both the enunciations and the proofs of these results. 
In all cases the condition with respect to the coefficients of the trigonometrical series considered is not merely sufficient for its purpose, but necessary if convergence of the type considered in an interval is required. I have not thought it desirable to go at length into the more general theorems which arise when convergence at a point only is demanded: in this case the condition $n^{-p} a_{n} \rightarrow 0, n^{-p} b_{n} \rightarrow 0$ may be replaced by the less stringent requirement

$$
n^{-p}\left\{a_{n} \cos n x+b_{n} \sin n x\right\} \rightarrow 0 \quad(n \rightarrow \infty) .
$$

11. Our present theory may be applied to the investigation of the behaviour of a power series on its circle of convergence, in the case when the coefficients tend towards zero. We shall assume the radius of convergence to be unity.

Let us denote the power series by

$$
\begin{aligned}
\sum_{1}^{\infty} c_{n} z^{n} & =\sum_{1}\left(a_{n}-i b_{n}\right) z^{n}=\sum_{1}^{\infty} c_{n} r^{n} e^{i n \theta} \\
& =\sum_{1}^{\infty} r^{n}\left\{a_{n} \cos n \theta+b_{n} \sin n \theta\right\}-i \sum_{1}^{\infty} r^{n}\left\{b_{n} \cos n \theta-a_{n} \sin n \theta\right\},
\end{aligned}
$$

where

$$
\begin{gathered}
c_{n} \rightarrow 0 \quad(n \rightarrow \infty), \\
a_{n} \rightarrow 0, \quad b_{n} \rightarrow 0 \quad(n \rightarrow \infty) .
\end{gathered}
$$

that is,

We have now to apply our theory simultaneously to a series

$$
\sum_{1}^{\infty} r^{n}\left\{a_{n} \cos n \theta+b_{n} \sin n \theta\right\},
$$

and to its allied series

$$
\sum_{1}^{\infty} r^{n}\left\{b_{n} \cos n \theta-a_{n} \sin n \theta\right\} .
$$

12. From Theorem 3 we have therefore at once the following:-

Theorem 5.-If a power series $\sum_{1}^{\infty} c_{n} z^{n}$ for which $c_{n} \rightarrow 0(n \rightarrow \infty)$, is such that its second integrated series, which necessarily converges uniformly on the circle of convergence (of radius unity), has for sum a function of $\theta$ whose second differential coefficient is a continuous function of bounded variation* for all points of an arc, then the power series converges uniformly throughout that arc.

\footnotetext{
* That is, $\phi_{1}(\theta)+i \phi_{2}(\theta)$, where $\phi_{1}$ and $\phi_{2}$ are continuous and of bounded variation.
} 
1917.] Restricted Fourier series and convergence of poWer series. 365 .

13. Hence we get almost immediately the next theorem.

TheOREm.-If the sum-function of a power series $\cdot \stackrel{1}{1}_{n}^{\infty} z^{n}$, for which $c_{n} \rightarrow 0(n \rightarrow \infty)$, has a unique limiting value $f(\theta)$ at each point $\theta$ of an arc $(\alpha, \beta)$ of the circle of convergence, and if $f(\theta)$ is then a function of bounded variation, the power series converges uniformly on that arc.

F'or, if

$$
f(r, \theta)=\sum_{n=1}^{\infty} c_{n} r^{n} e^{i n \theta} \quad(r<1)
$$

then, by hypothesis,

$$
\operatorname{Lt}_{\left(\mapsto 1, \theta \rightarrow \theta_{0}\right)} f(r, \theta)=f\left(\theta_{0}\right) \quad\left(\alpha<\theta_{0}<\beta\right) .
$$

Since this convergence to the limit $f\left(\theta_{0}\right)$ is uniform in $(r, \theta), f\left(\theta_{\theta}\right)$ is continuous, and we may integrate twice with respect to $\theta$, and get

$$
\operatorname{Lt}_{r \rightarrow 1} \int_{a}^{\theta} d \theta \int_{a}^{\theta} f(r, \theta) d \theta=\int_{a}^{\theta} d \theta \int_{a}^{\theta} f(\theta) d \theta,
$$

that is, $\quad \operatorname{Lt}_{r \rightarrow 1}\left(-\sum_{n=1}^{\infty} n^{-2} c_{n} r^{n} e^{i n \theta}\right\}=\int_{a}^{\theta} d \theta \int_{a}^{\theta} f(\theta) d \theta$.

But since the series on the left converges uniformly, the sum is a continuous fuuction of the ensemble $(r, \theta)$; this gives

$$
-\sum_{n=1}^{\infty} n^{-2} c_{n} e^{i n \theta}=\int_{a}^{\theta} d \theta \int_{a}^{\theta} f(\theta) d \theta,
$$

whence, by the preceding theorem, the required result at once follows.

14. As a very special case of the preceding, we get Fatou's theorem :Cor. (Fatou's Theorem). $-A$ power series $\sum_{n=1}^{\infty} c_{n} z^{n}$, for which $c_{n} \rightarrow 0$ $n \rightarrow \infty)$, converges uniformly on any arc of the circle of convergence, provided that, at every point of the arc, the series is regular.

In fact, if $\phi(z)$ be the analytic function represented by the power series $\sum_{1}^{\infty} c_{n} z^{n}$ inside the unit circle as circle of convergence, $\phi(z)$ is a continuous. function of $z$ with a continuous differential coefficient, not only inside the unit circle, but also on the given arc $(\alpha, \beta)$, since, by hypothesis, the series may be analytically continued over the arc $(\alpha, \beta)$. Thus, if, with the notation of the preceding article, we write

$$
\phi(z)=f(r, \theta) \quad(x<1),
$$


366 Restricted Fourier series and converaence of power series.

and on the arc $(\alpha, \beta), \quad \phi\left(z_{0}\right)=f\left(\theta_{0}\right)$,

we have

$$
f\left(\theta_{0}\right)=\operatorname{Lt}_{\left(r \rightarrow 1, \theta \rightarrow \theta_{0}\right)} f(r, \theta),
$$

and $f(\theta)$ is an integral with respect to $\theta$. Thus the conditione of the theorem are satisfied, whence the required result immediately follows.*

15. From Theorem 4 we get corresponding results in the theory of power series, the condition $c_{n} \rightarrow 0$ being replaced by $n^{-p} c_{n} \rightarrow 0(n \rightarrow \infty)$, and ordinary convergence being changed into Cesiro convergence $(C p)$. Similarly, from $\$ 10$, we get further results of the same kind respecting the fractional Cesàro convergence of a power series.

- Similar generalisations hold good for Marcel Riesz's extensions of Fatou's theorem. 